\title{
Actions symplectiques de groupes compacts
}

\section{Yves Benoist}

Published online: 30 June 2007

(C) Springer Science+Business Media B.V. 2007

\section{Erratum to: Geom Dedicata DOI 10.1023/A:1014253511289}

Corrections to "Action Symplectiques de groupes compacts"

The main aim of my paper [1] is an extension of the Guillemin-Sternberg-Kirwan convexity theorem to any symplectic action of a compact group on a compact symplectic manifold $(M, \omega)$. As an application, I deduce in chapter 6, extending Delzant's work, the description of all coisotropic actions of a torus $T$ on $M$, i.e. actions with at least one coisotropic orbit, up to isomorphism where isomorphism means equivariant symplectomorphism. But as Duistermaat and Pelayo discovered in [2], my conclusion for this application is too strong: the general coisotropic action is not a product of a Hamiltonian one by an anhamiltonian one, as I claimed in Proposition 6.17 and Theorem 6.6.A of [1], but a "fiber bundle" of hamiltonian over anhamiltonian. The aim of this text is to explain exactly where this omission occurs and how to correct it.

Note that this omission does not affect our convexity Theorem (Theorem 4.1 of [1]) which is the main result of [1]

Let $T$ be a torus, $(M, \omega)$ be a compact connected symplectic manifold endowed with a coisotropic effective action of $T$.

Recall from Corollary 6.16 of [1] that there exist a torus decomposition $T=T_{h} \times T_{a}$, an hamiltonian coisotropic action of $T_{h}$ on a compact simply-connected symplectic manifold $\left(M_{h}, \omega_{h}\right)$ and a coisotropic action of $\widetilde{T}_{a}$ by translation on a symplectic vector space $\widetilde{M}_{a}$ such that the universal cover $\widetilde{M}$ can be equivariantly and symplectically identified with the product $M_{h} \times \widetilde{M}_{a}$. Let us denote $\breve{T}:=T_{h} \times \widetilde{T}_{a}$ and $\Gamma:=\pi_{1}(M)$. Both groups are subgroups of the $\operatorname{group} \operatorname{Aut}(\tilde{M}):=\{\check{T}$-equivariant symplectomorphisms of $\tilde{M}\}$.

The online version of the original article can be found under doi:10.1023/A:1014253511289.

Y. Benoist $(\bowtie)$

Ecole Normale Supérieure-CNRS, 45 rue d’Ulm, 75230 Paris, France

e-mail: benoist@dma.ens.fr 
Recall also that according to Lemma 6.18 of [1], the elements of $\operatorname{Aut}(\tilde{M})$ permute the fibers of the projections $\widetilde{M} \simeq M_{h} \times \widetilde{M}_{a} \rightarrow \widetilde{M}_{a}$ hence induce automorphisms of $\widetilde{M}_{a}$.

Proposition 6.17 and Theorem 6.6.A must be replaced by the following statements, using freely the notations of [1] :

Proposition There exists a connected nilpotent Lie group $N$ which is a subgroup of $\operatorname{Aut}(\tilde{M})$ containing $\check{T}$ and $\Gamma$, such that the quotient $N / \Gamma$ is compact and such that the induced action of the quotient group $N_{a}:=N / T_{h}$ on $\widetilde{M}_{a}$ is simply transitive.

Let $\Gamma_{a}$ be the image of $\Gamma$ in $N_{a}$ and $M_{a}:=\widetilde{M}_{a} / \Gamma_{a} \simeq N_{a} / \Gamma_{a}$. In a less precise way (less precise because it does not describe completely the symplectic structure on the fibration), one can reformulate this proposition:

Any coisotropic action of a torus $T$ on a compact connected symplectic manifold $(M, \omega)$ is a fibration of the hamiltonian action of a torus $T_{h}$ on a symplectic manifold $M_{h}$ over an anhamiltonian action of a torus $T_{a}$ on a symplectic nilmanifold $M_{a}$.

Proof of Proposition The proof of this corrected version of Proposition 6.17 needs almost no changes: I will just explain these changes. The omission in the proof of Proposition 6.17 of [1] is on line 18 where I write: Autrement dit les fonctions $g_{\delta, \delta^{\prime}}$ ne dépendent pas de la première variable. In fact these functions $g_{\delta, \delta^{\prime}}$ may depend in an affine way in the first variable with a slope in $\mathfrak{t}_{h, \mathbb{Z}}$ because I had forgotten the "modulo $\mathfrak{t}_{h, \mathbb{Z}}$ " in the differentials $d g_{\delta, \delta^{\prime}}$. Hence these functions $g_{\delta, \delta^{\prime}}$ are in $\operatorname{Pol}\left(\mathfrak{l}^{*}\right)$ instead of $\operatorname{Pol}\left(\mathfrak{l}_{a}^{*}\right)$. Once this omission is taken into account, the proof of this new proposition is almost the same: the functions $f_{\delta}^{\prime}$ are in $\operatorname{Pol}\left(\mathfrak{l}^{*}\right)$ instead of $\operatorname{Pol}\left(\mathfrak{l}_{a}^{*}\right)$ and, after conjugating by an element $g$ in $\operatorname{Aut}(\tilde{M})$, the group $\Gamma$ becomes a subgroup of the connected nilpotent Lie group

$$
N_{p}:=\left\{[d f, s, \lambda] / f \in \operatorname{Pol}\left(\mathfrak{l}^{*}\right), d^{\circ} f \leq p, s \in \mathfrak{s}, \lambda \in \mathfrak{l}_{a}^{*}\right\} .
$$

for some $p \geq 1$. This group is not simply connected anymore but $N_{p} / T_{h}$ is. This allows to end the proof in the same way.

Remark Theorem 6.6.B of [1] needs no changes since, in this case $\mathfrak{t}_{h, \mathbb{Z}}=0$.

- As explained in Exercise 6.3 of [1], $N$ is either abelian or two-step nilpotent.

- Explicit examples where this fibered action is not a product have been discovered by Duistermaat and Pelayo in [2]. I thank them for sending [2] to me. In fact, the aim of [2] is to give a complete list of invariants for all coisotropic actions of tori. Such a classification can also be obtained using the cohomological approach of the proof of Proposition 6.17 of [1].

\section{References}

1. Benoist, Y.: Actions symplectiques de groupes compacts. Geom. Ded. 89, 181-245 (2002)

2. Duistermaat, J., Pelayo, A.: Symplectic torus actions with coisotropic principal orbit. arXiv:math.DG/0511676, 1-66 